\title{
Effects of texture and shape on perceived time to passage: Knowing "what" influences judging "when"
}

\author{
JOAN LÓPEZ-MOLINER \\ Universitat de Barcelona, Barcelona, Catalonia, Spain
}

AND

Eli Brenner and Jeroen B. J. Smeets

Erasmus MC, Rotterdam, The Netherlands

\begin{abstract}
In the present study, we examined whether it is easier to judge when an object will pass one's head if the object's surface is textured. There are three reasons to suspect that this might be so: First, the additional (local) optic flow may help one judge the rate of expansion and the angular velocity more reliably. Second, local deformations related to the change in angle between the object and the observer could help track the object's position along its path. Third, more reliable judgments of the object's shape could help separate global expansion caused by changes in distance from expansion due to changes in the angle between the object and the observer. We can distinguish among these three reasons by comparing performance for textured and uniform spheres and disks. Moving objects were displayed for $0.5-0.7 \mathrm{sec}$. Subjects had to decide whether the object would pass them before or after a beep that was presented $1 \mathrm{sec}$ after the object started moving. Subjects were not more precise with textured objects. When the disk rotated in order to compensate for the orientation-related contraction that its image would otherwise undergo during its motion, it appeared to arrive later, despite the fact that this strategy increases the global rate of expansion. We argue that this is because the expected deformation of the object's image during its motion is considered when time to passage is judged. Therefore, the most important role for texture in everyday judgments of time to passage is probably that it helps one judge the object's shape and thereby estimate how its image will deform as it moves.
\end{abstract}

Estimating the time that an object will take to reach an observer is important for many daily tasks (e.g., intercepting or hitting balls during some sports, avoiding collisions when we navigate). If the object of interest - for example, a ball - is moving straight toward the observer's eyes, its image expands isotropically during the whole trajectory. In such cases, optical variables can provide reliable information about time to contact. For example, the ratio $(\tau)$ between the visual angle $\theta$ and the rate of expansion $d \theta / d t$ of the approaching object's image signals the correct time to contact for an object moving at constant speed. Several authors have suggested that people use this ratio in various daily tasks (e.g., Lee \& Lishman, 1977; Lee, Young, Reddish, Lough, \& Clayton, 1983; Todd, 1981). Several variations on this ratio have proven to be relevant for judging time to contact as well (see, e.g., López-Moliner \& Bonnet, 2002; Rushton \& Wann, 1999; Smith, Flach, Dittman, \& Stanard, 2001). However, we do not interact only with objects that are traveling at a constant speed in a head-on approach, and we certainly do not always "interact" with them with our forehead. For instance, we often catch objects that would pass us if they were not caught some distance from the eyes. Thus, it seems reasonable to think that we often need to know how much time an object will take to pass arbitrary points of interest in space. Timeto-passage (TTP) studies aim at dealing with this kind of situation. For practical reasons, TTP is often defined in the literature (sometimes implicitly) as the time it will take an object to pass an imaginary vertical plane that passes through the observer's eyes (e.g., Kaiser \& Mowafy, 1993; Regan, 2002). This is what we will do here as well.

When an object is not moving straight toward the observer's eyes, TTP is not related to the relative rate of expansion in as straightforward a manner as is time to contact. Several more complicated optical measures specify the moment at which the object will reach a fixed position. These measures make use of additional information about angular velocity and binocular vision (e.g., Bootsma \& Craig, 2002; Lee, Georgopoulos, Clark, Craig, \& Port, 2001; Peper, Bootsma, Mestre, \& Bakker, 1994; Regan, 2002; Tresilian, 1991, 1993).

If the object that is going to pass the observer is not a sphere, its image is likely to undergo deformations (i.e., not to expand isotropically), making expansion a less 


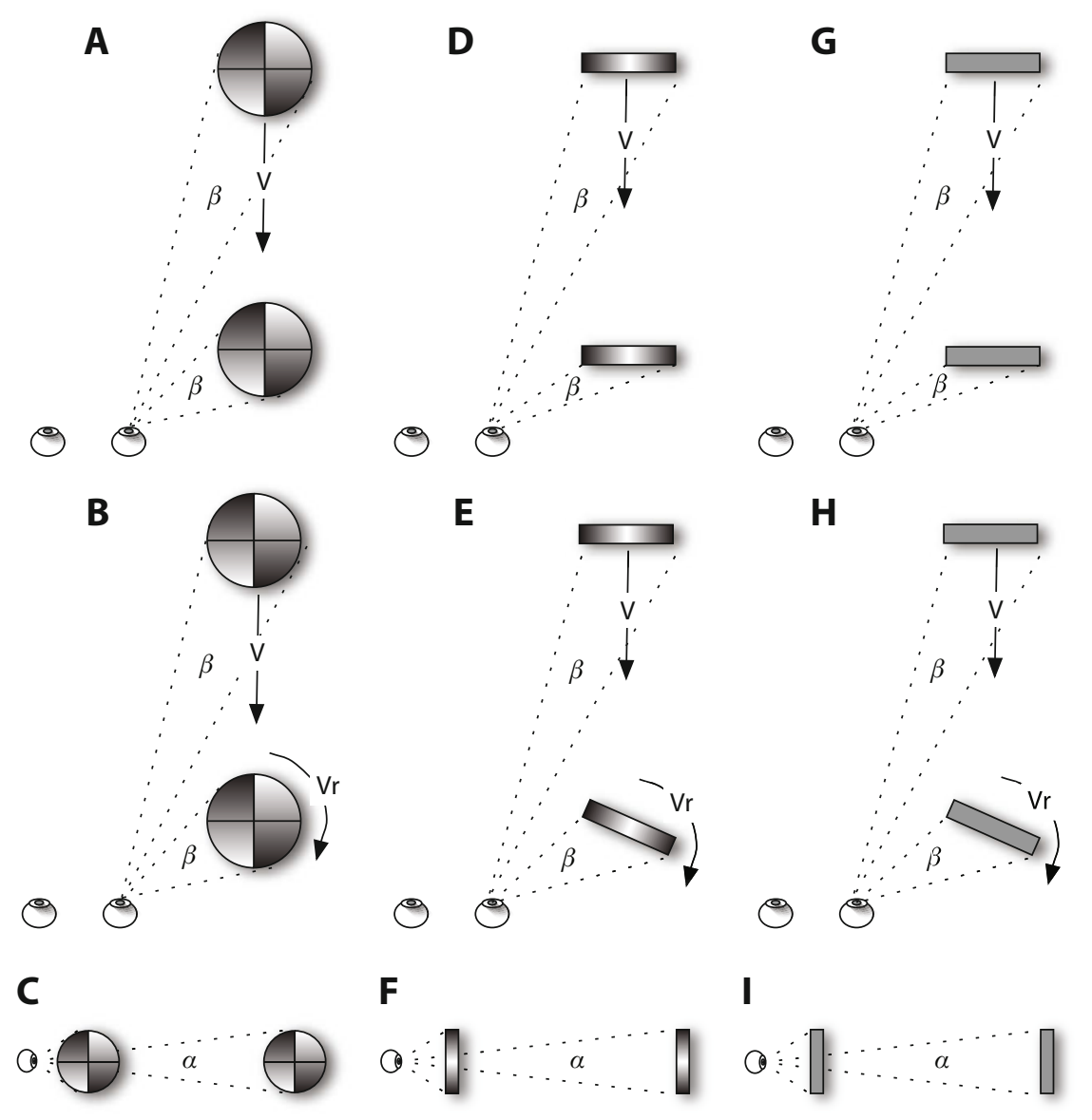

Figure 1. Sketches of the different conditions used in Experiment 1. The top (A, D, and G) and middle $(B, E$, and $H)$ rows of panels show top views of the nonrotating and rotating objects, respectively. The bottom row of panels $(C, F$, and I) shows side views (which are the same for rotating and nonrotating objects) and are on a smaller scale. The three columns show the three kinds of objects: textured spheres (left), textured disks (middle), and uniform disks (right). $\mathrm{V}$, velocity of approach to the plane of the observer; $\mathrm{Vr}$, rotational velocity.

straightforward variable for estimating the rate of change in distance (e.g., Gray \& Regan, 2000). Most studies have considered measures based on the outline of the object in question (usually a sphere). Here, we examine whether motion of texture within the object and knowledge of the object's shape (in which texture may play an important role) are important for judging TTP. In the following sections, we will introduce three possible ways in which texture might contribute to estimating TTP.

\section{Adding Local Expansion}

Consider the case of a textured sphere that is going to pass you (see Figure 1A). In principle, optical specification of the time remaining until the sphere reaches a fixed point is available in the global optic flow (Kaiser \& Mowafy, 1993; Tresilian 1991, 1993). This specification has generally been assumed to be determined by the image outline. However, if the object's surface is textured, texture elements can provide an estimate of the TTP of that part of the sphere (local $\tau_{1}$; see Tresilian, 1991). Having expansion within the outline as well as expansion of the outline will stimulate more (looming) detectors and could thereby give rise to more precise judgments. Previous studies on how the visual system integrates local motion signals (e.g., Morrone, Burr, \& Vaina, 1995) leave open such possibilities to obtain a better estimate of the rate of expansion. Indeed, Vincent and Regan (1997) showed that time to contact was underestimated when texture expanded at a higher rate than the outline. However, this applied to motion on a collision course, and the objects they used did not correspond to real rigid objects. Note that different rates of expansion can arise within rigid objects; for instance, the relative rate of expansion of visible texture elements at the front of an approaching rigid sphere is higher than that of the outline, because these elements are nearer to the observer. Whether or not veridical local expansion improves judgments of TTP is therefore still an open question.

\section{Motion From Changes in Relative Orientation}

A second way in which texture could be used to judge TTP can be illustrated by considering a car that is going 
to pass you. As it approaches, you see different parts of the car: When it is far away, you see mainly the front, but later on you see more and more of the side. Similarly, for the sphere in Figure 1A, the shift of texture elements relative to the outline could help one judge the sphere's displacement. The rate at which the view changes (as is evident from the shift of the texture within the outline) is related to the motion of the sphere (assuming that the sphere is not rotating). Thus, a shift of texture elements could provide additional independent information about the object's motion.

Li and Laurent (1995) examined whether the rate at which texture is occluded as a ball rolls toward the subject has an effect on time to contact. Note that this is a very different issue from the one studied here, because it specifically relies on the ball's rotating in space as it rolls across the ground surface to facilitate judgment of the object's velocity. Li and Laurent found that, whereas the time at which an action was initiated was not affected by the rate of texture occlusion, the velocity of the action was.

\section{Knowing the Shape}

The third way in which texture could help one judge TTP is less direct: Using texture to better judge an object's shape may help get rid of errors that arise from changes in the orientation of the target relative to the line of sight (as discussed in a different context in the previous section). The image of the sphere illustrated in Figure 1A expands isotropically. The horizontal and vertical projections of the retinal image (angles $\beta$ and $\alpha$ ) expand at the same rate (see Figure 1A and the corresponding side view in Figure 1C). In this case, TTP could be estimated by combining expansion with angular velocity (Bootsma \& Oudejans, 1993; Gray \& Sieffert, 2005). However, when the rate of expansion is not isotropic, matters are more complicated. Consider, for example, a disk oriented in the frontal plane that is going to pass an observer (Figure 1D). As it approaches the observer, the disk's image deforms. At some time, its horizontal extent (angle $\beta$ ) even shrinks, whereas its vertical extent (angle $\alpha$ ) expands (see Figures 1D and 1G and the corresponding side views in Figures $1 \mathrm{~F}$ and 1I). This results in a dramatically lower rate of expansion of the area of the projected image for a disk than for a ball, and could therefore give rise to an overestimation of the remaining time for a disk. If we know the object's shape, we can anticipate such effects. Do our expectations about the optic flow affect our judgments of when an object is going to pass us? An alternative would be to use only the expansion in the direction orthogonal to the object's motion (Tresilian, 1991). (In our examples, that would be the vertical expansion.) Finding that the expected deformation influences judgments of TTP would imply that texture could help judge TTP by providing information about the shape of the approaching object.

\section{Manipulating the Optic Flow}

The influence of changes in orientation on the perceived velocity of approach has been studied before (Gray \& Regan, 2000; Li \& Laurent, 1995; Scott, Li, \& Davids,
1996; Scott, van der Kamp, Savelsbergh, \& Oudejans, 2004). However, this always involved rotations of the objects in space. When such rotations reduced the global change in retinal image size, the objects appeared to be approaching more slowly. The reduction of image expansion in the situation shown in Figure 1D is completely different in that it is intrinsic to the path: It is expected if the object is not rotating.

In order to evaluate the three above-mentioned ways in which texture could help one judge TTP (local expansion, relative orientation, and knowing shape), we compared judgments for textured and uniform objects that either did or did not rotate as they moved. When they rotated, the rotation was such that the same part of the object remained visible (when the object was a sphere) or the outline did not change (when the object was a disk). Note that in the latter case the lateral contraction is eliminated, which could be taken to indicate that the disk is not approaching very fast; nevertheless, the rate of expansion of its area increases, which could be taken to indicate that it is approaching faster. Thus, if adding texture increases the resolution of estimates of important parameters such as the rate of expansion, we expect similar but more precise judgments for textured than for uniform objects. If changes in relative orientation contribute to the judgment, we expect both rotating disks and rotating spheres to appear to arrive later than ones that are not rotating, and that judgments will be more precise for nonrotating textured spheres than for rotating ones. If knowing the shape is essential to interpreting the expansion of the outline, then the velocity of textured rotating disks may be underestimated whereas that of uniform ones is not, because the texture indicates that we are dealing with a disk, and we therefore expect lateral contraction. The uniform rotating disk is equivalent to a uniform sphere, which would not be expected to contract laterally.

\section{EXPERIMENT 1}

\section{Method}

Subjects. Nine students of the University of Barcelona took part in the experiment. All had normal or corrected-to-normal vision and were naive with respect to the aims of the experiment. None of the subjects was stereo blind (StereoFly test, Stereo Optical Co.).

Stimuli and Apparatus. Stimuli were displayed on a Phillips 22-in. monitor (Brilliance 202P4) at a refresh rate of $118 \mathrm{~Hz}$ and a screen resolution of 1,154 $\times 864$ pixels. A 3Dlabs VP870 video card controlled the stereo shutter spectacles (CristalEyes). Simulated targets moved orthogonally to the frontal plane along a line that passed $150 \mathrm{~mm}$ to the right of the midpoint between the subject's eyes. The midpoint between the eyes was aligned with the left edge of the monitor in order to increase the visual field on the right. Naturally, the images were also rendered in the correct perspective for viewing from this position. The screen was $1 \mathrm{~m}$ from the subject's eyes.

The stimuli conformed to one of the six conditions shown in Figure 1. These conditions resulted from the use of three simulated objects, each of which could either rotate or not. The three objects were (1) a textured sphere (panels A, B, and C), (2) a textured disk (panels D, E, and F), and (3) a uniform disk (panels G, H, and I). The radius of all the simulated objects was $15 \mathrm{~mm}$. This simulated radius subtended an initial angle within the range of $1.33^{\circ}-2.20^{\circ}$ and a final angle within the range of $2.72^{\circ}-6.9^{\circ}$. 
Each of the three objects was presented either rotating around its vertical axis in a way that canceled the horizontal contraction of the disks' images and texture shift within the spheres' outlines (Figure 1, panels $\mathrm{B}, \mathrm{E}$, and $\mathrm{H}$ ) or without such rotation (panels $\mathrm{A}, \mathrm{D}$, and $\mathrm{G}$ ).

In order to ensure that the subjects used the judged time to contact rather than some other correlated measure, we combined various simulated velocities and starting distances. The simulated velocity could be $0.76,0.83,0.91,1.0,1.10,1.20$, or $1.32 \mathrm{~m} / \mathrm{sec}$. The simulated initial distance could be $0.78,0.85,0.93,1.0,1.09,1.18$, or $1.29 \mathrm{~m}$. When these seven velocities and seven distances are combined, 24 of the 49 combinations arrive in less than $1 \mathrm{sec}, 24$ arrive in more than $1 \mathrm{sec}$, and 1 arrives in exactly $1 \mathrm{sec}$. The shortest TTP was $0.594 \mathrm{sec}$, and the longest was $1.7 \mathrm{sec}$. The TTP was defined with respect to the front of the object (not its center) when the object was a sphere, but with respect to the center of the object, rather than the first edge to cross the plane through the subject's eyes, when the object was a (rotating) disk. The total number of distinct stimuli was 294 [7 (velocities) $\times 7$ (initial distances) $\times 6$ (texture combinations) $]$.

Procedure. Each simulated object was rotating when it appeared at its initial distance. It continued to rotate randomly about three orthogonal axes for $3 \mathrm{sec}$. The purpose of this rotation was twofold: first, to make sure that the subject had a clear idea of the shape of the object, and, second, to give the subject time to fixate the object. After $3 \mathrm{sec}$, the object started approaching the observer at the designated constant velocity. If the object was a disk, the rotation was planned so that it would always be oriented in the frontal plane at the moment that it started approaching. During the approach, there was no random rotation. The object remained visible for a random presentation time between 0.5 and $0.7 \mathrm{sec}$. Like Gray and Regan (1998), we used an auditory beep to designate the reference time. This beep was always presented exactly $1 \mathrm{sec}$ after the object started approaching. The subject was instructed to press one of two buttons to indicate whether the front (and not the center) of the object had passed the eye plane before or after the reference beep was sounded. In each block, the subjects were shown all 294 stimuli in random order. Each subject took part in three blocks. At the beginning of each block, the subject practiced for 40 trials, selected at random from the 294 possibilities. Feedback was provided after each of these trials.

Analysis. We fit a logistic curve with two parameters (PSS and $S D$ ) to the proportion of "after-the-beep" responses as a function of TTP, as follows:

$$
P=\frac{1}{1+\exp [(\mathrm{PSS}-t) / S D]} .
$$

$P$ is the probability of an "after-the-beep" response given a value $t$ of the simulated TTP. PSS is the point of subjective simultaneity- that is, the TTP value that elicits 50\% "after" responses. A larger PSS (curves shifted to the right) means that the object appears to arrive earlier (fewer "after" responses) than simulated. $S D$ is inversely related to the slope of the function. A fit of Equation 1 to the data points averaged across subjects gives a general impression of the data. In order to quantify the reliability of the results, we performed paired $t$ tests on the parameters PSS and SD that were obtained by fitting Equation 1 to each individual subject's data.

\section{Results and Discussion}

Figure 2 shows the results of Experiment 1. Figure 2A shows the proportion of "after" responses as a function of TTP with the best fit for each condition. Figures $2 \mathrm{~B}$ and $2 \mathrm{C}$ show the mean PSS and $S D$, respectively, averaged across subjects, for each of the six conditions. The $S D$ values were similar for all the conditions $(0.12 \mathrm{sec})$ and consistent with values from previous studies (e.g., Gray \& Regan, 2000; Regan \& Hamstra, 1993). They are not much greater than values for synchronizing a flash with a beep (Brenner, van Beers, Rotman, \& Smeets, 2006), so subjects appear to be able to do the extrapolation quite well. Adding texture did not improve the sensitivity of TTP judgments. However, the PSS was different in some conditions (in agreement with findings reported in Gray \& Regan, 2000).

As we conducted multiple $t$ test comparisons, we used a corrected critical alpha value $(\alpha=.02)$ that was obtained by applying the false discovery rate method (Benjamini \& Hochberg, 1995). The rotation that cancels the contraction of the outline made textured disks appear to arrive later [in paired $t$ tests comparing rotating and nonrotating objects, $t(8)=2.8, p=.02]$. The corresponding difference was even stronger for uniform disks, but in that case the effect was not significant $[t(8)=2.3, p=.05]$. The difference between the two rotating disks (Figures $1 \mathrm{E}$ and $1 \mathrm{H}$ ) was also not significant $[t(8)=1.3, p=.22]$. Note that the larger image expansion that occurred when the disk rotated made the disk appear to arrive later, which is the opposite of what one would predict for the use of a global measure of expansion (or $\tau$ ).

A possible explanation for the systematic effect of rotating the disks is that our subjects did not consider the center of the objects as the part that should cross them. The right side will pass the reference plane earlier than the center of the disk for rotating disks, but not for nonrotating disks. The time difference between the TTP for the side and for the center is, on average, about $12 \mathrm{msec}$. In our analysis, we used the TTP of the center of the disk. Obviously, the subjects did not use the front part of the rotating disk, because, if they had, the disk would have appeared to arrive earlier (larger PSS) for rotating disks. However, even basing judgments on the rear part of the rotating disk cannot explain the observed difference of $51 \mathrm{msec}$. Rotating disks were also perceived to arrive about $56 \mathrm{msec}$ later than spheres $[t(8)=-4.85, p<.001]$. It is unlikely that this is because judgments were based on the wrong part of the sphere (e.g., the center rather than the front), because nonrotating disks were perceived to arrive in approximately the same amount of time as spheres $[t(8)=$ $-0.791, p=.43]$.

The shading of the symbols in Figure 2A indicates the simulated velocity. The correlation between simulated velocity and TTP is evident: The darker symbols (greater velocities) are further to the left (smaller TTP). However, it is also clear that velocity alone cannot account for the subjects' performance: Within the symbols for a given velocity, we see systematic differences in the proportions of "after" responses for different values of TTP. Moreover, the correlation between simulated velocity (or initial distance) and TTP that arises from our independently varying velocity and initial distance obviously cannot account for the differences between the conditions. In our interpretation of the PSS data, we rely on the differences between the conditions, because other systematic errors could have many origins that are irrelevant for our questions, such as subjects' misjudgment of the moment of the tone relative to that of the visual stimulus, or of the position of the reference plane.

In summary, this experiment shows that adding texture does not automatically make detection more precise; it did not increase the slopes of the psychometric functions (Fig- 


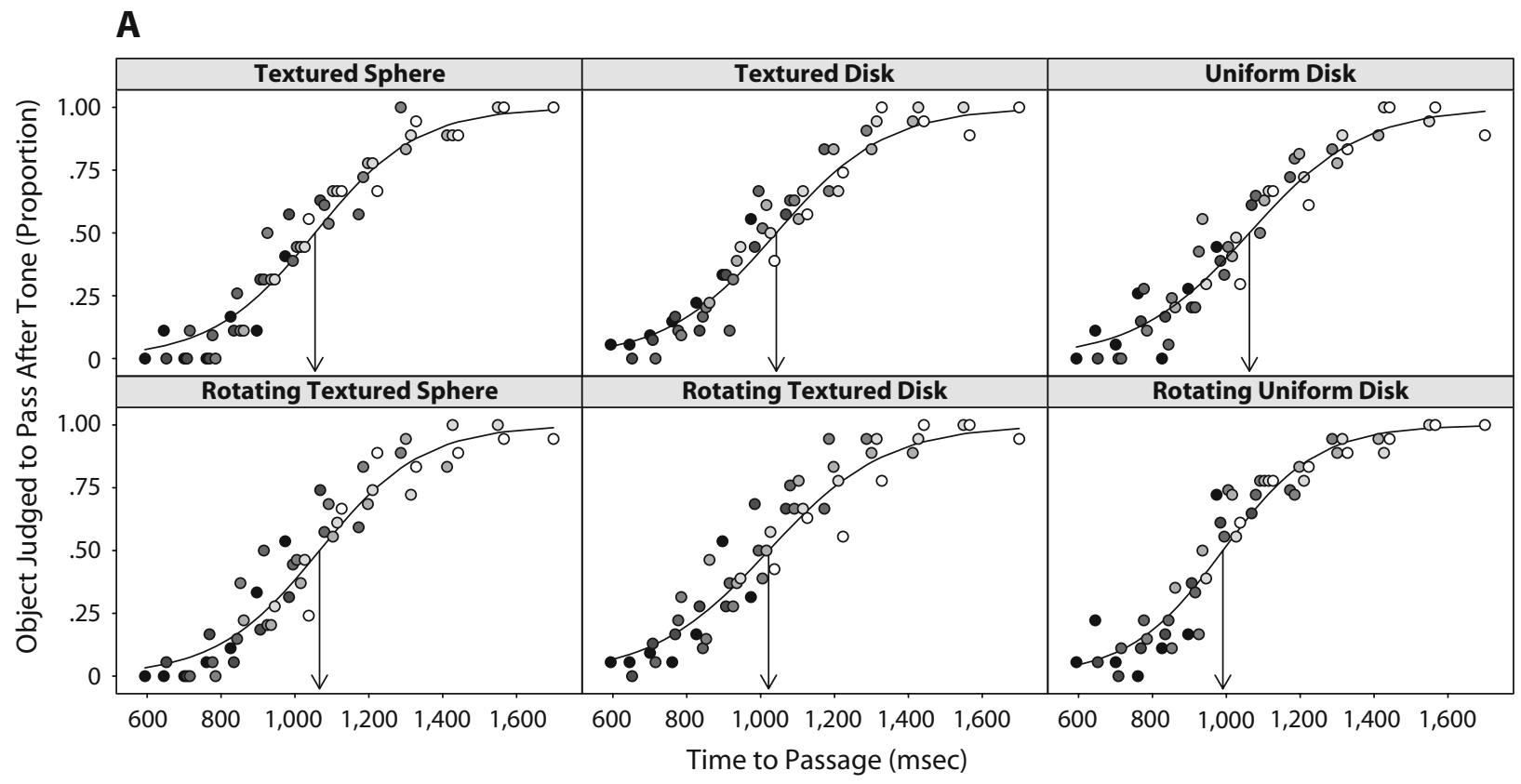

B

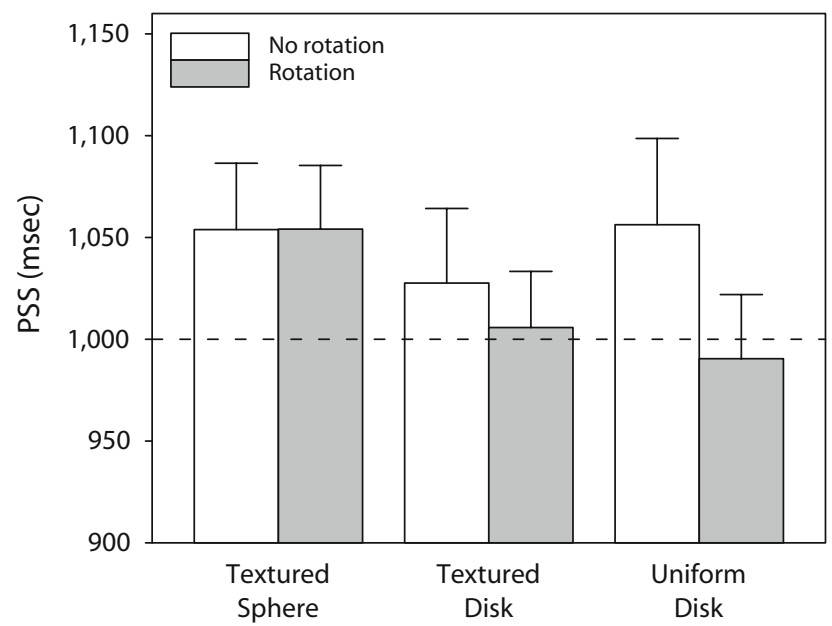

C

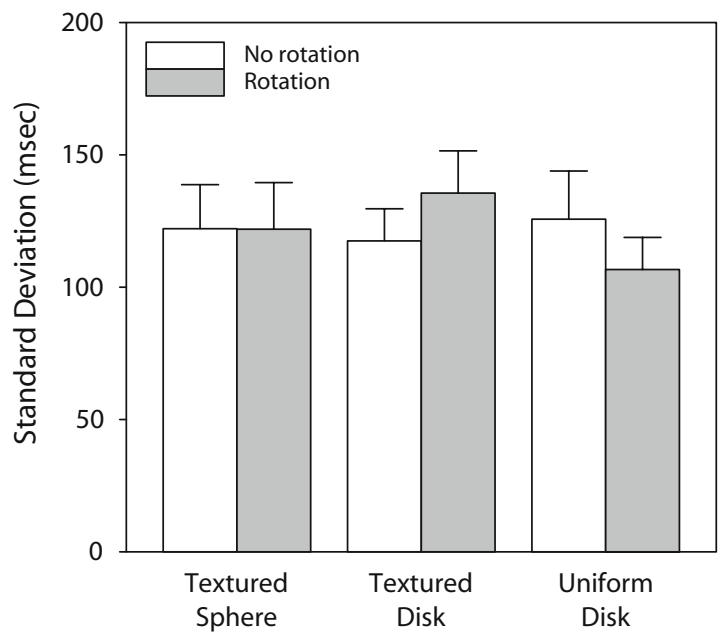

Figure 2. Results of Experiment 1. (A) Proportion of trials on which the object is judged to have passed after the tone was sounded, as a function of the simulated time to passage for each of the six conditions. Data points are averages across all 9 subjects. The curve denotes the best fit of a logistic function. Arrows indicate the simulated point of subjective simultaneity (PSS), or the time to passage at which the fit suggests that subjects are as likely to judge the object to have passed after the tone as to judge it to have passed before the tone. (B) Average of the values of PSS derived from separate fits to each subject's data. Note that larger values indicate that the target is judged to arrive earlier than it really does. The dashed line indicates a value of 1 sec, corresponding to veridical judgments. (C) Average of the standard deviations estimated from the separate fits. The error bars show the between-subjects variability, expressed as the standard error of the mean.

ures $2 \mathrm{~A}$ and $2 \mathrm{C}$ ). Thus, we find no evidence of judgments improving because more receptors are stimulated. Rotating the (textured) spheres also had no effect, suggesting that the changing view of the object is not used to estimate TTP - at least not under these conditions, in which objects sometimes do rotate. However, the subjects did not rely only on global expansion; if they had, we would expect rotating disks to appear to arrive earlier than nonrotating ones (or at the same time if only vertical expansion is considered). The fact that rotating disks appeared to arrive later suggests that it is not the expansion itself that is critical, but the expansion in relation to one's expectations. This is particularly evident from a comparison of the uniform rotating disks and textured spheres, because, in terms of the changing outline, simulating a uniform rotating disk is equivalent to simulating a uniform sphere. That is why we did not include uniform spheres in our simulation.

\section{EXPERIMENT 2}

To further test the hypothesis that the way in which changes in the retinal image are interpreted to estimate the 
TTP depends on the changes that one expects on the basis of the target's shape, we conducted a second experiment, in which we compared rotating uniform disks with uniform spheres. These objects give rise to practically identical retinal images during the motion. Thus, subjects could differentiate between the two images only as they rotated before starting to move. If subjects were to respond differently to these two stimuli, we would have definitive evidence of an influence of the subject's expectations.

\section{Method}

Subjects. Ten students of the University of Barcelona took part in the second experiment. None of them had taken part in Experiment 1. All had normal (or corrected) vision and were naive with respect to the aims of the experiment.

Stimuli and Apparatus. The only difference between Experiments 1 and 2 was the kind of objects that were simulated. The same approach velocities and the same initial distances were assigned to three kinds of targets: a disk, a rotating disk, and a sphere. None of them was textured. The first and second conditions were identical to the corresponding conditions in Experiment 1. The uniform sphere was new. The total number of distinct stimuli was $147(7 \times 7 \times 3)$.

Procedure. As in the first experiment, the simulated object rotated for $3 \mathrm{sec}$ around three orthogonal axes before starting to move. The subjects were told that only two kinds of uniform objects could appear and that the initial rotation had the purpose of letting them know the type of shape the objects would have. When the object was a disk, they saw this clearly as it rotated before starting move. The subjects realized that they would see no rotation when the object was a sphere. Except for the fact that each subject took part in six blocks instead of three, the procedure was exactly the same as in the previous experiment. Each of the 147 stimuli was presented once within each block.

Hypothesis testing. Since the rotating disk and the sphere generate the same images as they approach, any strategy based only on optical variables during the movement will give the same results for these two conditions. On the other hand, if the expected deformation of the optic flow is taken into account (because knowledge of the shape contributes to interpreting the optic flow), performance for the uniform sphere may be more like that for the disk that is not rotating. Since we know from the first experiment that judgments for the rotating and nonrotating disks differ, this comparison should reveal whether or not prior knowledge of the shape is relevant.

\section{Results and Discussion}

The data analysis was identical to that of Experiment 1. Individual fits allowed us to obtain the PSS and $S D$ for each subject. Figure 3A shows the distribution of "after" responses as a function of the simulated TTP, and the curves denote the best fit of Equation 1. The means of the PSS and SD across subjects are shown in Figures 3B and $3 \mathrm{C}$, respectively. In general, the PSS was larger than that observed in Experiment 1 (1,098 vs. $1,031 \mathrm{msec} ; t=$ $-3.14, p=.003)$. The average $S D$ was similar to that in Experiment 1 (110 vs. $120 \mathrm{msec} ; t=0.93, p=.35)$. As before, we corrected for multiple comparisons, and the new alpha value was set to .03 . The difference between the judgments for the rotating and nonrotating (uniform) disks was smaller than that in Experiment 1, but again, the rotating uniform disk was perceived to arrive significantly later than the nonrotating disk $[t(9)=4.02, p=.003]$.

The TTP judgments for the sphere were clearly more like the judgments for the disk that was not rotating than like those for the rotating disk. In fact, the uniform disk that was not rotating was even perceived to arrive $18 \mathrm{msec}$ later (lower PSS) than the uniform sphere $[t(9)=-2.57$, $p=.03]$, whereas it was perceived to arrive earlier than the rotating disk. Although the images that were presented during the motion were almost identical for the rotating disk and the uniform sphere, the rotating disk was perceived to arrive $46 \mathrm{msec}$ later than the uniform sphere $[t(9)=-3.98, p=.003]$. The images were not exactly the same, but the differences were so small that the first author (maximum score of $40^{\prime \prime}$ on the StereoFly test) performed at chance level when trying to discriminate between the uniform sphere and the rotating uniform disk during the motion. Moreover, a preliminary experiment (published in abstract form by López-Moliner, Brenner, \& Smeets, 2004) showed that when there was no preexposure and subjects were not told that the target was a sphere, a uniform sphere was judged to arrive significantly later than a textured one, as we found for the rotating uniform disk in comparison with the textured or uniform sphere in Experiment 1.

These results demonstrate that the response is not based exclusively on the actual optic flow, but that the observed optic flow is compared with the expected flow. In other words, the subjects took into account the known shape of the approaching object. In our experiment, the shape was known from seeing the object rotate before it started approaching, but in daily life the shape will often be judged from the texture within the object.

\section{GENERAL DISCUSSION}

Other studies have borne upon the role of texture in judging time to contact (e.g., DeLucia, Kaiser, Bush, Meyer, \& Sweet, 2003; Li \& Laurent, 1995; Vincent \& Regan, 1997) - for instance, by examining whether adding conflicting texture expansion affects time-to-contact estimates as defined by changes in an object's outline. Vincent and Regan found that time to contact was overestimated when texture expanded at a lower rate than the outline (but see DeLucia et al., 2003), showing that not only the outline is considered. This is not a completely unrealistic condition, because different rates of expansion do normally occur within a single object if the object is extended in depth, because nearer parts of the object will reach the observer earlier. Thus, the fact that we found no improvement in judgments of TTP when we added texture does not necessarily mean that only changes in the outline are relevant. Perhaps the resolution with which the rate of expansion can be determined is not critical for performance on this task (e.g., it may be negligible in comparison with the poor temporal resolution; Brenner at al., 2006), so that providing more expanding structures does not improve precision.

The second mechanism we proposed specifically relies on the object's not rotating: It uses the rate at which the view of the object changes to judge the object's velocity. The simplest version of this proposal could be rejected, because it predicts that our rotating spheres would appear to arrive later (lower values of PSS), whereas they were actually perceived to arrive at the same time as nonrotating 


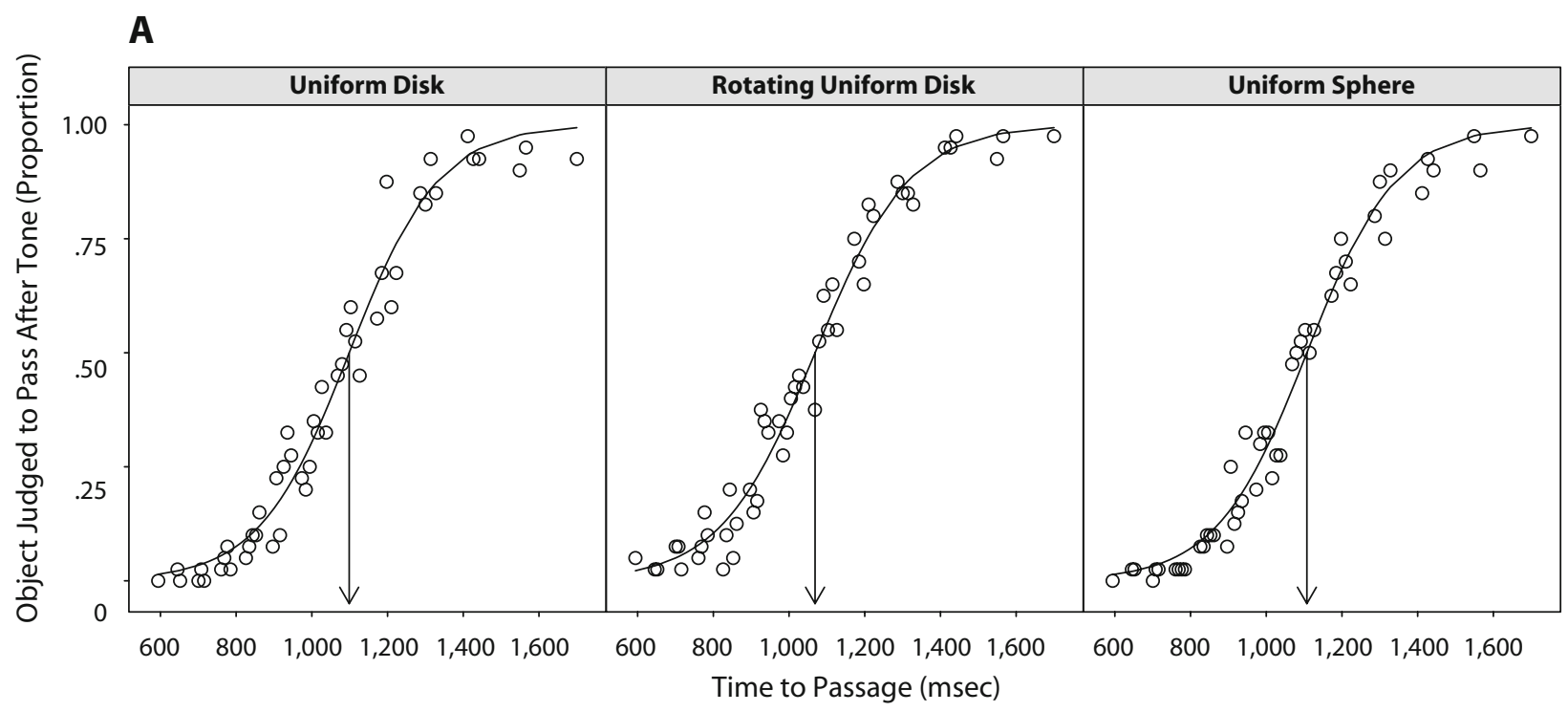

B

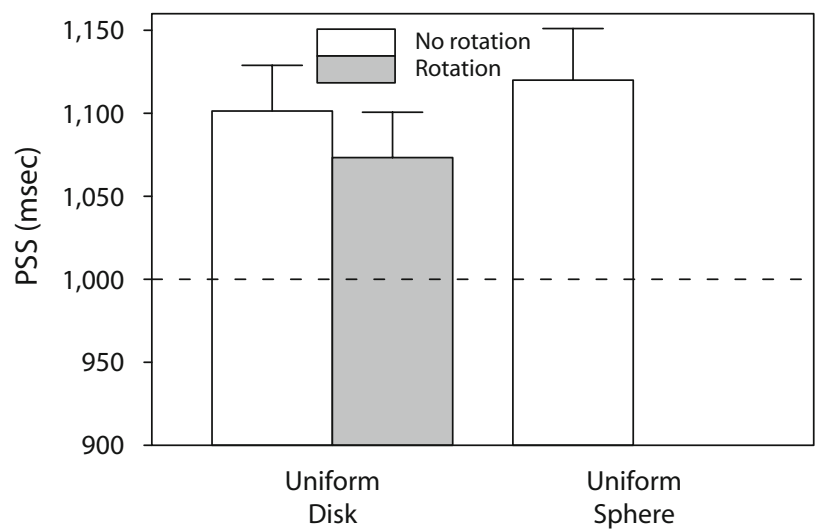

C

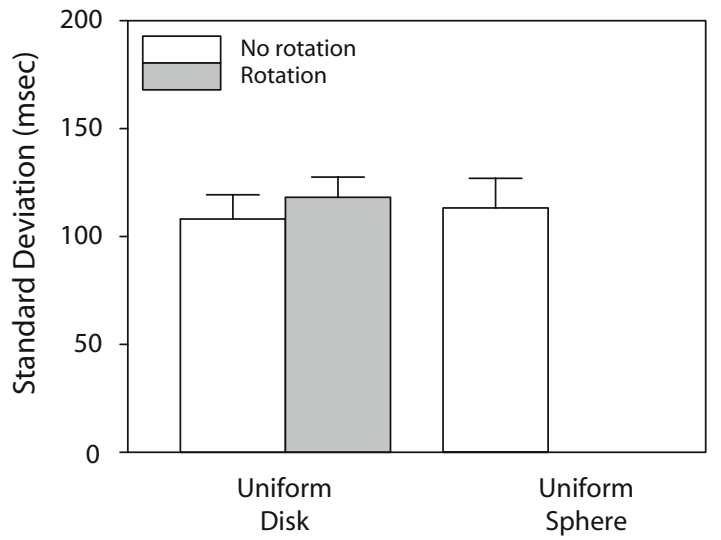

Figure 3. Results of Experiment 2. (A) Proportion of trials on which the object is judged to have passed after the tone was sounded, as a function of the simulated time to passage for each of the three conditions. (B) Simulated time to passage at the point of subjective simultaneity. (C) Standard deviations. Data show averages across 10 subjects. For further details, see Figure 2.

spheres. However, the rotating disks did appear to arrive later than their nonrotating counterparts, despite the fact that the rotation increased the amount of expansion without systematically changing the lateral translation of the whole object or global binocular information about the object's motion. Thus, the deformation is not ignored. This new factor adds to previous ones that have been found to affect TTP judgments: familiar size (DeLucia, 2005) and image velocity (Kerzel, Hecht, \& Kim, 1999).

In our second experiment, we saw that the (lack of) deformation is interpreted in relation to what the subject expects (our third proposal). Thus, although the changing view of the texture within a target is probably not used to judge TTP, expected changes in the outline probably are. Note that subjects do not simply scale the expansion in relation to what they expect, because if they had done so they would have judged the rotating disks to arrive sooner, not later. Thus, changes in the object's orientation relative to the observer during the movement (assuming that the object is not rotating) do appear to be considered when judging TTP. The willingness to assume that the object is not rotating must be quite strong, because it seems to persist even when there is clear visual evidence that the object is rotating, as in the case of our (textured) disks or the objects in the studies of Gray and Regan (2000) and Scott et al. (1996). This suggests that TTP judgments rely more on a global comparison between actual and expected flow than on a detailed analysis of the flow itself. Since the texture on an object's surface is an important source of information about its shape, both directly and indirectly through binocular disparities, texture probably influences estimates of TTP mainly through its contribution to judgments of the shape of the object in question.

\section{AUTHOR NOTE}

We thank John Flach and two anonymous reviewers for their comments and suggestions. We also thank Jordi Escoda for running the experiments. E.B. and J.B.J.S. are now at the Vrije Universiteit Amsterdam. Correspondence concerning this article should be addressed to J. López-Moliner, Grup de Recerca Neurociència Cognitiva, Departa- 
ment de Psicologia Bàsica, Universitat de Barcelona, P. Vall d'Hebron, 171, 08035 Barcelona, Spain (e-mail: j.lopezmoliner@ub.edu).

\section{REFERENCES}

Benjamini, Y., \& Hochberg, Y. (1995). Controlling the false discovery rate: A practical and powerful approach to multiple testing. Journal of the Royal Statistical Society: Series B, 51, 289-300.

Bootsma, R. J., \& Craig, C. M. (2002). Global and local contributions to the optical specification of time to contact: Observer sensitivity to composite tau. Perception, 31, 901-924.

Bootsma, R. J., \& Oudejans, R. R. (1993). Visual information about time-to-collision between two objects. Journal of Experimental Psychology: Human Perception \& Performance, 19, 1041-1052.

Brenner, E., van Beers, R. J., Rotman, G., \& Smeets, J. B. J. (2006). The role of uncertainty in the systematic spatial mislocalization of moving objects. Journal of Experimental Psychology: Human Perception \& Performance, 32, 811-825.

DeLucia, P. R. (2005). Does binocular disparity or familiar size override effects of relative size on judgements of time to contact? Quarterly Journal of Experimental Psychology, 58A, 865-886.

Delucia, P. [R.], Kaiser, M. K., Bush, J. M., Meyer, L. E., \& Sweet, B. T. (2003). Information integration in judgements of time to contact. Quarterly Journal of Experimental Psychology, 56A, 1165-1189.

Gray, R., \& Regan, D. (1998). Accuracy of estimating time to collision using binocular and monocular information. Vision Research, 38, 499-512.

Gray, R., \& REgan, D. (2000). Estimating the time to collision with a rotating nonspherical object. Vision Research, 40, 49-63.

Gray, R., \& SiEfFERT, R. (2005). Different strategies for using motionin-depth information in catching. Journal of Experimental Psychology: Human Perception \& Performance, 31, 1004-1022.

KaISER, M. K., \& Mowafy, L. (1993). Optical specification of time-topassage: Observers' sensitivity to global tau. Journal of Experimental Psychology: Human Perception \& Performance, 19, 1028-1040.

Kerzel, D., Hecht, H., \& Kim, N.-G. (1999). Image velocity, not tau, explains arrival-time judgments from global optical flow. Journal of Experimental Psychology: Human Perception \& Performance, 25, 1540-1555.

Lee, D. N., Georgopoulos, A. P., Clark, M. J. O., Craig, C. M., \& Port, N. L. (2001). Guiding contact by coupling the taus of gaps. Experimental Brain Research, 139, 151-159.

LEe, D. N., \& Lishman, R. (1977). Visual control of locomotion. Scandinavian Journal of Psychology, 18, 224-230.

Lee, D. N., Young, D. S., Reddish, P. E., Lough, S., \& Clayton, T. M. H. (1983). Visual timing in hitting an accelerating ball. Quarterly Journal of Experimental Psychology, 35A, 333-346.
LI, F.-X., \& LAURENT, M. (1995). Occlusion rate of ball texture as a source of velocity information. Perceptual \& Motor Skills, 81, 871-880.

López-Moliner, J., \& Bonnet, C. (2002). Speed of response initiation in a time-to-contact discrimination task reflects the use of $\eta$. Vision Research, 42, 2419-2430.

López-Moliner, J., Brenner, E., \& Smeets, J. B. J. (2004). The role of texture in judging time-to-contact [Abstract]. Perception, 33(Suppl.), 20.

Morrone, M. C., Burr, D. C., \& Vaina, L. M. (1995). Two stages of visual processing for radial and circular motion. Nature, 376, 507-509.

Peper, L., Bootsma, R. J., Mestre, D. R., \& Bakker, F. C. (1994). Catching balls: How to get the hand to the right place at the right time. Journal of Experimental Psychology: Human Perception \& Performance, 20, 591-612.

REGAN, D. (2002). Binocular information about time to collision and time to passage. Vision Research, 42, 2479-2484.

Regan, D., \& Hamstra, S. J. (1993). Dissociation of discrimination thresholds for time to contact and for rate of angular expansion. Vision Research, 33, 447-462.

Rushton, S. K., \& WANN J. P. (1999). Weighted combination of size and disparity: A computational model for timing a ball catch. Nature Neuroscience, 2, 186-190.

ScotT, M. A., LI, F.-X., \& Davids, K. (1996). The shape of things to come: Effects of object shape and rotation on the pickup of local tau Ecological Psychology, 8, 343-352.

Scott, M. A., van der Kamp, J., Savelsbergh, G. J. P., \& Oudejans, R. R. D. (2004). Object rotation effects on the timing of a hitting action. Research Quarterly for Exercise \& Sport, 75, 130-137.

Smith, M. R., Flach, J. M., Dittman, S. M., \& Stanard, T. (2001) Monocular optical constraints on collision control. Journal of Experimental Psychology: Human Perception \& Performance, 27, 395-410.

ToDD, J. T. (1981). Visual information about moving objects. Journal of Experimental Psychology: Human Perception \& Performance, 7 , 795-810

Tresilian, J. R. (1991). Empirical and theoretical issues in the perception of time to contact. Journal of Experimental Psychology: Human Perception \& Performance, 17, 865-876.

Tresilian, J. R. (1993). Four questions of time to contact: A critical examination of research on interceptive timing. Perception, 22, 653680.

Vincent, A., \& RegAn, D. (1997). Judging the time to collision with a simulated textured object: Effect of mismatching rate of expansion of object size and of texture element size. Perception \& Psychophysics, 59, 32-36.

(Manuscript received March 17, 2005;

revision accepted for publication December 15, 2006.) 